Bull. Mater. Sci., Vol. 8, No. 5, December 1986. pp. 581-586. (C) Printed in India.

\title{
Optical black coatings for space applications
}

\author{
K RAMASESHAN, M VISWANATHAN and G K M THUTUPALLI \\ ISRO Satellite Centre, Bangalore 560017 , India \\ MS received 19 October 1985; revised 2 January 1986
}

\begin{abstract}
The durability properties of PTX-205 optical black coatings deposited on metallic and non-metallic substrates are studied. The coatings are stable against adverse environmental requirements for space applications. Typically, coatings with 8-11 $\mu$ thickness are opaque and have an average reflectance loss $(<5 \%)$ in the spectral range of $200-1500 \mathrm{~nm}$. The absorption to emission ratio is 1.01 .
\end{abstract}

Keywords. Black coatings; opaque coatings.

\section{Introduction}

Electrically conducting optical black coatings are extensively used in various terrestrial and space-oriented optical, thermal and electrical appliances (Birnbaum et al 1982). A few important applications among these are: (i) in high absorbing and low scattering of optical devices in far infrared cryogenic systems; (ii) as special masks in satellite-based electro-optical sensors; (iii) in thermal detectors and solar absorbers to increase their efficiency; and (iv) in space-borne systems to keep the differential charging of spacecraft external surfaces and equipment to a minimum to prevent problems due to electrostatic fields, arc etc.

For these, a variety of optical black coatings ranging from simple carbon soot layers of burning camphor, wax, kerosene etc., to vacuum-evaporated metallic and non-metallic blacks are available (Harris et al 1948; Okanto and Hatanaka 1981). However, when these coatings are used for defence and space applications, they should not only meet the basic optical and electrical properties needed for terrestrial systems, but also should functionally be stable against the adverse environmental conditions (table 1) to which they are exposed to, over an extended period of time. But, our experience shows that the majority of these black coatings cannot meet these requirements.

Even among the limited black paints available, there are hardly any studies available on their durability properties although their optical and electrical properties are known. In many cases, the details of the process technology are kept as guarded trade secrets.

Keeping these in view, a systematic study of the functional and durability properties of various organic and inorganic blacks has been carried out and in this paper, the coating process technology, the functional and durable properties of PTX-205 of Polytronix brand are reported (Back 1982).

\section{Process technology}

The various performance requirements and specifications to be met for a useful space/terrestrial borne black paint are summarized in tables 1 and 2 . While some of 
Table 1. Performance specifications.

\begin{tabular}{ll}
\hline $\begin{array}{l}\text { Adhesion } \\
\text { Abrasion }\end{array}$ & $\begin{array}{l}\text { Cellotape peel test (MIL-F-48616) } \\
\text { Eumidity }\end{array}$ \\
Eraser abrasion for 50 strokes with $0.5 \mathrm{~kg}$ continuously applied. \\
Steady state $\left(48^{\circ} \pm 2^{\circ} \mathrm{C}\right)$ and $95-100 \%$ relative humidity for \\
$24 \mathrm{hr}(\mathrm{MIL}-\mathrm{C}-675 \mathrm{~A})$
\end{tabular}

Table 2. Performance specifications.

\begin{tabular}{ll}
\hline Parameter & Desired value \\
\hline Substrate for coating & Metallic/non-metallic \\
Outgassing (total mass loss) & Less than $1 \%$ \\
Pinholes on the coated area & Class-A (number of pinholes of size \\
& 0.05 mm per square cm will be less \\
& than 1). \\
Transmittance & $1 \%$ in the visible and near IR \\
Total reflectance & $<5 \%$ \\
Emissivity & $>0.8$ \\
\hline
\end{tabular}

the applications such as thermal control of devices require only deposition of black coatings on suitable metallic/non-metallic substrates, some other applications such as (space borne sensor masks etc) also require a suitable pattern generation on the black paints. Therefore, its optimized process technology is presented in this paper. The functional and durability properties of the black paint so deposited on metallic/ non-metallic substrates with and without pattern are also reported.

\subsection{Substrate preparation}

For non-metallic substrates, optically plane glass and quartz $(25 \mathrm{~mm} \times 25 \mathrm{~mm}$ size and $1.5 \mathrm{~mm}$ thick) are taken and their surface quality is better than $60: 40$ in the optical standards. Aluminium and copper foils $(25 \mathrm{~mm} \times 25 \mathrm{~mm}$ square and $100 \mu$ thick) and free from sleeks, scratches and wrinkles etc., are used for metallic substrates.

Substrate cleaning prior to deposition of coating plays a vital role and the following cleaning procedure is adopted. The glass and quartz substrates are vapourdegreased to remove grease, oil etc in reagent grade trichloroethylene, followed by a sequential ultrasonic cleaning in alkaline solution, distilled water and chromic acid. They are then rinsed in distilled water and finally, ultrasonically, cleaned in reagent 
grade isopropyl alcohol. The aluminium and copper foils are vapour-degreased and cleaned in dilute hydrochloric acid, distilled water and dried. The substrates are baked at $200^{\circ} \mathrm{C}$ for $30 \mathrm{~min}$.

\subsection{Coating process and generation of pattern}

The cleaned dry surface on which the black coating has to be deposited is treated with silane type adhesion promoter (Polytronix) and spin-dried at $4000 \mathrm{rpm}$. The black paint is then spread uniformly, spin-coated and baked at $90^{\circ} \mathrm{C}$ for $45 \mathrm{~min}$. The photoresist (Waycoat HPR-204 positive photoresist) is similarly spin-coated on the dried black surface and pre-baked at $90^{\circ}$ for $45 \mathrm{~min}$. The photoresist is exposed under the desired photomask, developed and post-baked at $90^{\circ} \mathrm{C}$ for $45 \mathrm{~min}$. The unprotected black is etched in a warm dilute alkaline solution at $50^{\circ} \mathrm{C}$ to realize the final pattern. The treated surface is rinsed in distilled water and dried. The undeveloped photoresist is stripped and the sample is baked at $200^{\circ} \mathrm{C}$ for $60 \mathrm{~min}$.

\subsection{Thickness measurement}

Thickness of black coatings is maintained in the range of $8-12 \mu$. Films with thickness lower than $8 \mu$ on the non-metallic substrates cannot meet the required opacity level. On the other hand, for films, thicker than $12 \mu$ on both metallic and non-metallic substrates, although the functional properties remain unaltered the adhesion is poor. The thickness is measured at various points randomly on the coatings using vertical metroscope (Carl Zeiss, Jena ABBE P 01) having a resolution of $0 \cdot 1 \mu$.

It has been observed that due to variations in the speed of the spinning system used in this Laboratory, a non-uniformity upto $\pm 0.5 \mu$ is noticed in the coatings. But as it can be seen later, these variations do not have adverse effect on the required properties of the coatings when subjected to environmental test conditions.

\section{Test results}

\subsection{Functional properties}

3.1a Thermo-optical properties: The transmission spectra of the coated samples are measured in the range of $400-1000 \mathrm{~nm}$ and 2-15 $\mu$ on spectrometers (Schimatzu Model VV-120-02 and Perkin Elmer Model 783). The reflection spectra are measured in the same ranges on the photospectrometer (Beckmann Model AC 7A MIV and Perkin Elmer Model 783). For non-metallic substrates, the average reflectance is $<5 \%$ in the entire spectral range and transmittance is $<0.1 \%$ in the range $400-1500 \mathrm{~nm}$ and zero in the range $2-15 \mu$. For metallic substrates, while the transmittance is zero, the reflectance values are less than $5 \%$ in the entire spectral range. Typical reflectance spectra on metallic/non-metallic substrates are shown in figures $1 \mathrm{a}$ and $1 \mathrm{~b}$. The noise spectrum in the range 6 to $7 \mu$ seen in figure $1 \mathrm{~b}$ is due to the ambient water vapour and is not the intrinsic property of the coatings.

The emissivity values are measured using an emissometer (Devices and Services 


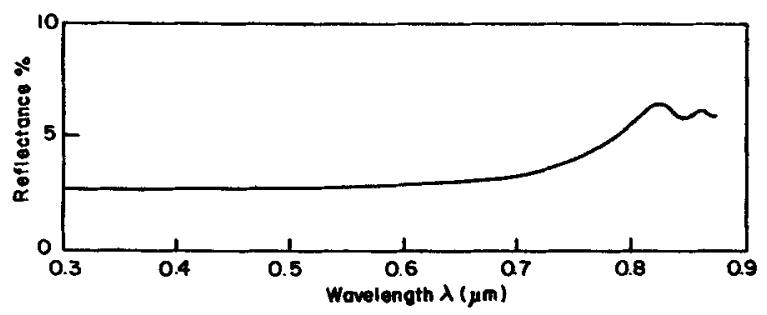

Figure 1a. Reflection spectra of PTX-205.

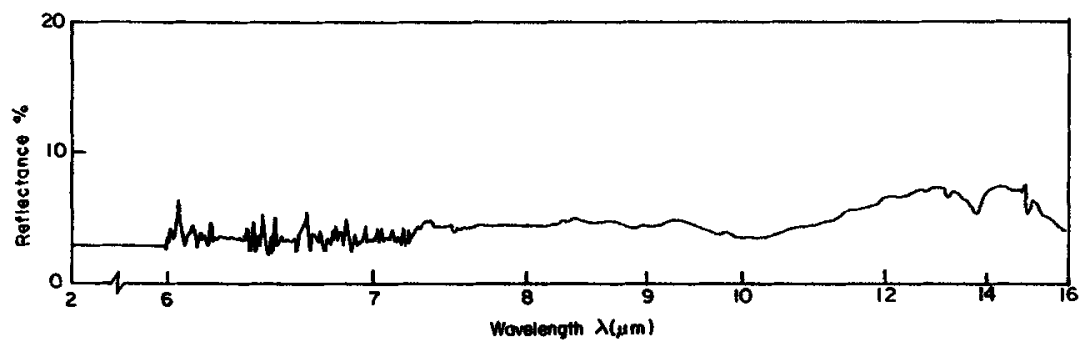

Figure 1b. Reflection spectra of PTX-205.

Company Model 4, E.S.No. 175) and the values range from 0.83 to 0.86 depending upon the surface finish. However, the results are reproducible to an accuracy of $\pm 1 \%$. The corresponding absorption to emission (a/e) ratio is calculated to be 1.01 .

3.1b Electrical properties: To estimate the volume and surface resistivities, the following simple method is adopted.

A conducting foil is cut and placed exactly over the painted area of a metallic substrate and compressed firmly against the paint surface. Resistance is measured from the top surface of the conducting foil to the bottom of the substrate. From this the volume resistivity is calculated. The measurements on different samples show that the volume resistivity is in the range of $18.14 \mathrm{ohm}$ metre to $6.1 \mathrm{ohm}$ metre for the coating thickness corresponding to the range of 10 to $8 \mu$. The surface resistivity is deduced from this and the corresponding values range from $1.814 \mathrm{ohms}$ per square to $0.76 \mathrm{ohms}$ per square.

3.1c Mechanical properties: Five samples are selected at random in a batch of 30 samples and the adhesion test is conducted according to military standards (MIL-F48616). A scotch tape (peel strength $0.5 \mathrm{~kg} / \mathrm{cm}$ ) is uniformly adhered to the coating surface by pressing it against the surface. The tape is peeled rapidly at an angle between $45^{\circ}$ and $90^{\circ}$ to the surface. The coating is evaluated for peel-off and other surface defects such as pin holes, scratches etc. Also, the optical properties are measured as post-adhesion test. The bonding is extremely good in all the samples and no deterioration in the optical properties and no defects in the coating surface quality are found. 
A moderate abrasion test is conducted on the same set of samples. The coated surface is abraded with a standard eraser mounted in an eraser abrasion counting tester. The rubbing is done across the surface from one point to another over the same path for 50 strokes with a load of $0.5 \mathrm{~kg}$ continuously applied. The length of the stroke is approximately 3 times the diameter of the eraser piece. The surface is evaluated for cosmetic defects and the optical properties measured as post-abrasion tests. No deterioration is observed suggesting the good abrasion resistance of the black paint system.

\subsection{Durability properties}

Stability of the functional properties of the samples, when subjected to the accelerated environmental conditions (table 1), is evaluated to ascertain the usefulness of the black coatings for various other applications in space. A batch of 30 samples are divided at random into sets, each consisting of 5 numbers, and tests are conducted on the set of samples in the sequence given in figure 2 . Commercially available environmental test chambers are used and the standard procedures are adopted (MIL-C-675A). The results are summarised in table 3.

\section{Conclusion}

The PTX-black coatings are stable functionally against adverse environmental conditions such as humidity, thermal shock cyclings, etc. These coatings can be

Table 3. Summary of test results under accelerated environmental conditions.

\begin{tabular}{|c|c|c|}
\hline \multicolumn{3}{|l|}{ Type of test } \\
\hline Adhesion & $\begin{array}{l}\text { Surface quality } \\
\text { Optical properties }\end{array}$ & $\begin{array}{l}\text {-Good } \\
\text {-Good }\end{array}$ \\
\hline Abrasion & \multicolumn{2}{|l|}{- do- } \\
\hline Humidity & \multicolumn{2}{|l|}{ do- } \\
\hline Thermal shock & \multicolumn{2}{|l|}{- do- } \\
\hline \multicolumn{3}{|l|}{ Thermal soaking } \\
\hline \multirow[t]{2}{*}{ (i) Hot soaking } & \multicolumn{2}{|c|}{$\begin{array}{l}\text { * Pinholes (5-10 number) appear randomly in, the } \\
\text { samples. But their sizes are }<0.05 \mathrm{~mm}\end{array}$} \\
\hline & \multicolumn{2}{|c|}{$\begin{array}{l}\text { The integrated optical transmission is less than } 0-1 \% \text { in } \\
\text { the visible and near IR }\end{array}$} \\
\hline (ii) Cold soaking & \multicolumn{2}{|c|}{$\begin{array}{l}\text { No change in the surface quality or pinhole size and } \\
\text { number }\end{array}$} \\
\hline \multirow[t]{2}{*}{ Vacuum baking } & \multicolumn{2}{|c|}{$\begin{array}{l}\text { *Pinholes appear randomly. But their size is }<0.05 \mathrm{~mm} \text {. } \\
\text { The integrated optical transmission is not affected and is } \\
<0.1 \% \text {. }\end{array}$} \\
\hline & \multicolumn{2}{|c|}{ Total mass loss $<1 \%$} \\
\hline Salt solubility & $\begin{array}{l}\text { Surface quality } \\
\text { Optical properties }\end{array}$ & $\begin{array}{l}\text {-Good } \\
\text {-Good }\end{array}$ \\
\hline
\end{tabular}

* (In hot soaking and vacuum baking, except for the vacuum environment, the temperature and duration of heating are the same. Therefore, with extended heating, the pinholes might appear due to the release of entrapped air lockets during coating) 


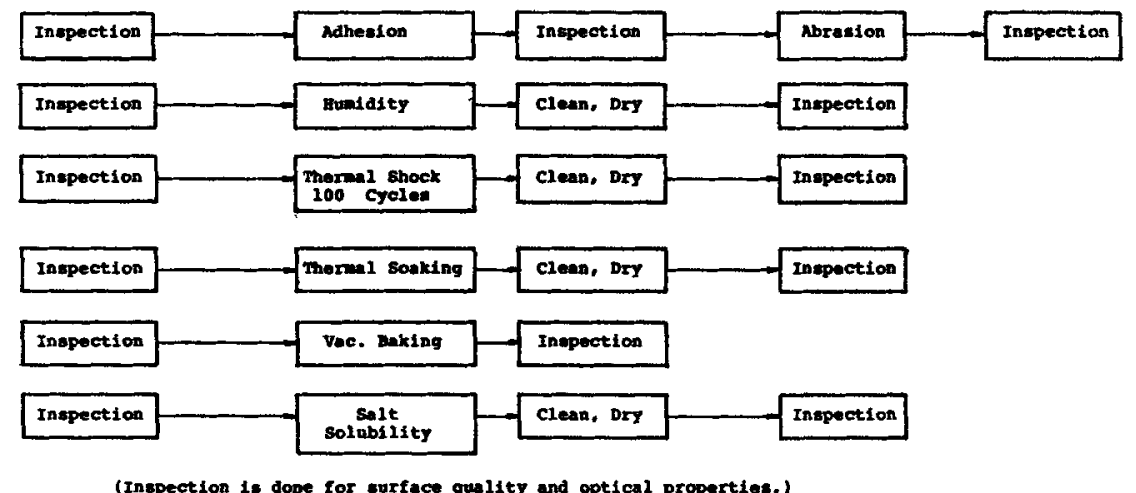

Figure 2. Test sequence.

applied on metallic/non-metallic substrates, either as a uniform layer or as layer with required pattern generation and these can find immense applications in various terrestrial and space-borne systems.

\section{References}

Birnbaum M, Mettler E C and Cieland E L 1982 Proceedings of SPIE 36260

Harris L, McGinnies R T and Siegel B M 1948 J. Opt. Soc. Am. 38582

Lineback R J 1982 Electronics April 2142

Okanto S and Hatanaka Y 1981 Bull. Bismuth Inst. 338

Polytronix data sheet P O Box 3024 Richardson Texas 75081

US Military Specification MIL-C-675A 1976 Coating of glass optical elements

US Military Specification MIL-F-48616 1977 Federal Specification L-T-90 Tape pressure sensitive adhesive cellophane and cellulose acetate 